\title{
Dysregulation of glucose transport and transporters in perfused hearts of genetically obese (fa/fa) rats
}

\author{
D. Zaninetti, R. Greco-Perotto, F. Assimacopoulos-Jeannet and B.Jeanrenaud \\ Laboratoires de Recherches Métaboliques, Faculty of Medicine, University of Geneva, Switzerland
}

\begin{abstract}
Summary. The regulation of glucose transport in normal and insulin-resistant obese rat hearts have been studied by measuring glucose transport via the efflux of labelled 3-0-methyl-Dglucose. Glucose transporters in obese rat hearts were also investigated using the labelled cytochalasin B-binding assay. Basal, and insulin - or increasing workload-induced stimulation of glucose transport was decreased in obese rat hearts compared to those of normal ones. Total number of glucose transporters (plasma membrane plus microsomal ones) was about half that previously reported for normal rat hearts. Insulin or workload favoured the translocation of glucose transporters from an intercellular pool (microsomes) to the plasma membrane, as they do in normal rats. Due to the measured decrease in total number of transporters of obese rat hearts, those present in the plasma membrane (under basal conditions, or following stimulation by insulin or workload) were
\end{abstract}

less than those previously found in normal rat hearts tested under identical conditions. In obese rat hearts, regulation of plasma membrane transporters was perturbed. The Hill coefficient (an index of positive cooperativity amongst glucose transporters) was paradoxically decreased by insulin while leaving affinity values unaltered. The Hill coefficient was unaltered by workload, although the affinity values were increased compared to respective controls. To sum up, obese rat hearts have decreased total transporter number, and although the two stimuli studied favour the translocation of available transporters, they fail to "activate" them adequately once present in the plasma membrane.

Key words: Perfused heart, genetically obese (fa/fa) rats, glucose transport, glucose transporters, cytochalasin B assay.
The existence of insulin resistance at the level of various muscles is well substantiated in several animal models of non-insulin-dependent diabetes and/or obesity [1]. It has been demonstrated to be present in vitro as well as in vivo (reviewed in 1), and bears on insulin receptor number [2]; insulin receptor autophosphylation [3]; glucose uptake [4, 5]; glycolysis [4-6] and glycogen synthesis [7].

With regard to glucose uptake, it has been shown that basal, as well as insulin-stimulated glucose uptake, was decreased in most obesity syndromes studied [2, 4-6]. As is now well established in adipocytes $[8,9]$ and also described in muscle [10], glucose transporting molecules (glucose transporters) are present both in the plasma and in some intracellular (microsomal) membranes. Furthermore, insulin has been shown [8-10] to favour the translocation of microsomal glucose transporters to the plasma membrane, thereby stimulating glucose uptake. Recently, our laboratories have further shown that, in normal brown adipose tissue [11] or perfused hearts from normal rats [12] insulin does not only favour the translocation of glucose transporters, but increases the "intrinsic activity" of the transporters once inserted in the plasma membrane. This was evidenced by the observation of two insulin-induced changes in glucose transport: an apparent increase in the affinity of the transporter for glucose and the occurrence of positive cooperativity [12]. This was reflected by changes in the properties of the glucose transporter toward cytochalasin B (increase in the affinity and cooperativity). It has also been shown that in the normal rat heart, the glucose transport, the translocation of glucose transporters and the changes in the functional properties of the latter, once in the plasma membrane, can be evoked not only by insulin but, in the absence of the hormone, by increasing the workload [13].

In adipose cells obtained from aging insulin-resistant rats [14], or from normal rats made insulin resistant by feeding them a high-fat/low carbohydrate diet [15], insulin-stimulated 3-0-methyl-D-glucose uptake was found to be reduced. In these insulin-resistant adipocytes, the translocation process elicited by insulin was operative, but the number of glucose transporters present in the plasma membrane under the influence of the hormone was reduced, due to a reduction in the total number of transporters (plasna plus microsomal ones) $[14,15]$. No data are as yet available for insulin-resistant muscle types. Due to this, the aim of the present study was to characterise the glucose transport in perfused 
hearts of genetically obese insulin-resistant ( $\mathrm{fa} / \mathrm{fa}$ ) rats, and to study the regulation of their glucose transporter systems. The data obtained were compared to those of lean rat hearts studied exactly at the same time and published in [13].

\section{Material and methods}

\section{Animals}

Fifteen-week-old normal $(\mathrm{Fa} / \mathrm{Fa})$ or genetically obese (fa/fa) male rats of the Zucker strain bred in our laboratory were used. They had free access to a standard laboratory chow (Lacta 299, Provimi-Lacta SA, Cossonay, Vaud, Switzerland) and were maintained at a constant temperature $\left(22^{\circ} \mathrm{C}\right)$ in an animal quarter with a $12 \mathrm{~h}$ artificial light cycle. Lean and obese rats were all studied concomitantly, i.e. at the same period of the same year. However, as many questions were initially posed regarding normal animals and published elsewhere [12, 13], comparisons between lean and obese animals, particularly when dealing with glucose transporters number and characteristics were made by referring to the previously published papers on lean rats.

\section{Heart perfusion and 3-0-methyl-D-glucose transport studies}

Rats were anesthetised with pentobarbital $\left(90 \mathrm{mg} \mathrm{kg}^{-1}\right)$. The heart was rapidly removed, placed in an ice-cold $0.9 \% \mathrm{NaCl}$ solution for $10 \mathrm{~s}$, cannulated via the aorta and perfused according to Langendorff as described elsewhere [5]. The perfusion medium consisted of a Krebs Ringer bicarbonate buffer, $\mathrm{pH} 7.4$, with pyruvate $(5 \mathrm{mmol} / \mathrm{l})$ as energy source. For measuring 3-0-methyl-D-glucose (3-0-M-glucose) transport, hearts were pre-perfused for $10 \mathrm{~min}$ with or without the stimuli defined below, with a recirculating medium containing $6 \mathrm{mmol} / 1(0.3 \mathrm{mCi} / 1){ }^{14} \mathrm{C}-3-0-\mathrm{M}$-glucose and $6 \mathrm{mmol} / 1(0.3 \mathrm{mCi} / \mathrm{l})$ ${ }^{3} \mathrm{H}$-L-glucose (loading period). Ten min were needed to reach steadystate levels of labelled substrates. Labelled L-glucose was used to calculate the extracellular and free diffusion components. At the end of the loading period, perfusion was continued without recirculation, while switching to a medium containing no sugar. Aliquots of the medium were then collected every $12 \mathrm{~s}$ in vials placed on a fraction collector (Gilson TDC 220, Villier le Bel, France). Each vial contained $12 \mathrm{~s}$ fractions of the medium with 3-0-M-glucose and L-glucose arising from the heart. 3-0-M-glucose was in the intra- and the extracellular space; L-glucose mainly in the extracellular and, to some extent (via simple diffusion), in the intracellular space. 3-0-M-glucose counter-transported by facilitated diffusion (efflux) could be calculated by subtracting, in each vial, total labelled L-glucose content from total labelled 3-0-M-glucose content as described elsewhere [5]. Unless stated otherwise, hearts were perfused at low pressure $(50 \mathrm{~mm} \mathrm{Hg})$. When basal 3-0-M-D-glucose efflux rates were investigated a pre-perfusion period of $10 \mathrm{~min}$ was allowed to presumably wash away most endogenous insulin. When the effect of insulin was measured, the hormone $(10 \mathrm{mU} / \mathrm{ml})$ was added to both pre- and perfusion media. The workload effect on glucose efflux rates was measured by raising the perfusion pressure (during the pre- and perfusion periods) from 50 to $100 \mathrm{~mm} \mathrm{Hg}$.

\section{Heart plasma and microsomal membrane preparation}

For membrane preparation, hearts of obese rats were perfused for 15 min in presence or in absence of stimulus indicated above. Hearts were then disconnected from the perfusion apparatus and immediately frozen in liquid nitrogen. The membrane preparations (respectively plasma and microsomal membranes) were then carried out as described elsewhere $[12,13]$.

\section{Enzyme marker assays}

Total proteins were measured by the Bio-Rad method, with gamma globulin as standard [16]. The 5'-nucleotidase was the marker used for plasma membranes and was determined as described elsewhere [17]. The NADPH cytochrome $\mathrm{c}$ reductase, a marker of microsomal membranes, was determined according to the method of Sottocasa et al. [18].

\section{Cytochalasin B-binding assays}

Cytochalasin B-binding was carried out on plasma and microsomal membranes obtained from obese rat hearts. As binding curves obtained with either 10 or 5 different ligand concentrations $(40-500 \mathrm{nmol} / \mathrm{1})$ resulted in identical results [12], the present experiments were made with only five ligand concentrations, using a modified technique described and validated previously [11-13,19]. As also described previously [11-13, 19], binding of labelled cytochalasin was carried out either in the presence of $250 \mathrm{mmol} / 1$ sucrose (i.e. specific + unspecific bindings) or in that of $500 \mathrm{mmol} / 1$ unlabelled glucose (non-specific binding). The differences between the two binding curves yielded the specific (i.e. D-glucose displaceable) cytochalasin B-binding [11-13, 19]. The Hill equation [20] was used to analyse the binding data. The plot of the linearised Hill equation, i.e. $\log$ $(\mathrm{b} / \mathrm{Ro}-\mathrm{b})=\mathrm{n} \log (\mathrm{F})-\log \left(\mathrm{K}^{\prime}\right)$ (in which $\mathrm{Ro}=$ maximal binding capacity; $b=$ bound cytochalasin $B ; F=$ free cytochalasin $B ; n=$ Hill coefficient; $\mathrm{K}^{\prime}=$ constant comprising interaction factor and intrinsic dissociation constant; $\mathrm{Kd}=\mathrm{K}^{1 / 1 / n}=$ dissociation constant) permitted to obtain the Hill coefficient $n$, the Ro value (maximal binding) and normalised Kd values; $n$ was the slope of the linearised Hill equation and $\mathrm{Kd}=10^{\log (\mathrm{F})}$, where $\log (\mathrm{b} / \mathrm{Ro}-\mathrm{b})=0$. Using the values of $\mathrm{n}, \mathrm{Kd}$ and Ro, the Hill curve was plotted (bound versus free) together with the data points. The affinity values $(\mathrm{Ka})$ were used in this paper and were $1 / \mathrm{Kd}$. In the present study, complete plots of data were not represented. Results were, for clarity sake, and due to the previously mentioned validations $[11-13,19]$, shown as table $(\mathrm{n}, \mathrm{Ka})$ and bar graphs (Ro).

\section{Statistical analysis}

Statistical analyses were performed with the two-tailed Student's t-test for unpaired data.

\section{Results}

\section{3-0-methyl-D-glucose transport}

The 3-0-methyl-D-glucose transport was measured by its efflux from preloaded hearts, as depicted by Figure 1. As may be seen, basal as well as insulinelicited glucose transport was reduced in obese rat hearts compared to those of lean control rat hearts. Similar data were obtained when using increased workload as the sole stimulator (Fig.1).

Insulin and workload increased glucose transport 10-11 fold in obese and 8-9 fold in lean rats.

\section{Glucose transporters as measured by the $\left[{ }^{3} \mathrm{H}\right]$ cytochalasin B-binding assay}

To determine the role of glucose transporters in the abnormal D-glucose transport of insulin-resistant obese rat hearts, $\left[{ }^{3} \mathrm{H}\right]$ cytochalasin B-binding assays were performed in plasma and microsomal membranes ob- 
tained from obese rat hearts. The D-glucose inhibitable cytochalasin binding assays were performed on enriched plasma and microsomal membranes. The latter had similar characteristics as those previously reported for normal heart [13], as depicted by Table 1 . The enrichment of plasma membranes was about 20 -fold, that of microsomal membranes was about 7 -fold. The measured specific cytochalasin B-binding sites were expressed as $\mathrm{pmol} / \mathrm{mg}$ of membrane protein and adjusted to those which would have been observed, had the membrane fractions been free of cross-contamination. Such adjustments were based on specific activities of the enzyme markers, with the assumption that 5'-nucleotidase activity was localised specifically in plasma membranes and that NADPH cytochrome $c$ reductase activity was specific for microsomal membrane. Each sample was always corrected for the cross-contamination, a contamination that was $7 \%$ plasma membranes in microsomes and 49\% microsomal membranes in the plasma ones. The cytochalasin B-binding curved obtained were similar to those previously described with membranes of normal hearts [13]. Specific binding was obtained by the difference between total and glucose displaceable binding and analysed according to Hill [20]. For clarity sake, and as mentioned in "Materials

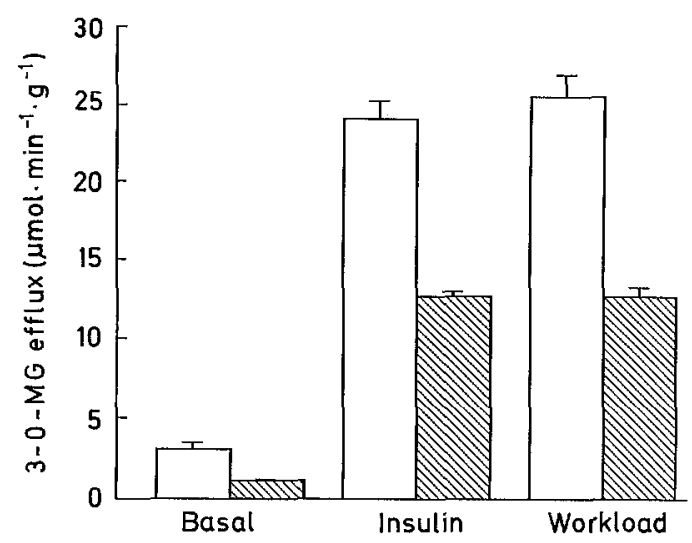

Fig. 1. Effect of insulin or increasing workload on 3-0-methyl-D-glucose (3-0-MG, $6 \mathrm{mmol} / \mathrm{l})$ transport by perfused hearts of lean (open bars $\square$ ) and obese (hatched bars $\mathbb{Q}$ ) rats. 3-0-MG transport was measured by its efflux from preloaded organ under basal conditions $(50 \mathrm{~mm} \mathrm{Hg}$ perfusion pressure); in the presence of a maximal insulin concentration $(10 \mathrm{mU} / \mathrm{ml})$; following increased workload achieved by raising perfusion pressure from 50 to $100 \mathrm{~mm} \mathrm{Hg}$. Values are means 3-5 experiments \pm SEM. Lean vs obese: all values statistically different at $2 p$ at least $<0.005$ and methods", the maximal binding (Ro) per membrane of obese rat heart (plasma or microsome), were represented as bar graphs (Fig.2-3). To enable comparison of these data with the published ones dealing with normal hearts studied contemporarily [13], dotted areas have been provided for the latter.

As shown by Figure 2, insulin added to obese rat hearts favoured the translocation of glucose transporters from microsomal to plasma membrane, without altering the total number of transporters (plasma + microsomal transporters). Insulin-induced translocation was evidenced by an increase of the number of transporters present in plasma membranes that occured concomitantly with a decrease of those located in microsomal membranes. However, as the total number of transporters was decreased by at least two-fold in obese rat heart compared to normal (Fig. 2, compare open and hatched bars to shaded areas), the number of transporters actually translocated into plasma membrane under the influence of insulin was reduced compared to normal rats.

The increase in workload (obtained by raising perfusion pressure from 50 to $100 \mathrm{~mm} \mathrm{Hg}$ ) had an insulin-like effect upon the redistribution of the glucose transporters between plasma and microsomal membranes. Indeed, as shown by Figure 3, the appearance of transporters in the plasma membranes increased while decreasing in microsomal membranes. Similary to what was observed before, the total number of transporters was decreased by at least two-fold in obese heart compared to normal [13], so that the number of transporters translocated to the plasma membrane under the influence of increased workload was reduced compared to normal rats.

Upon computing transporter number per mg protein, marker enzyme recovery and protein content per gramme of heart the total number of transporters per gramme of obese rat heart could be calculated and was, respectively (means of 3-8 individual experiments) $505 \pm 49$ (basal conditions); $573 \pm 38$ (insulin); $608 \pm 48$ (workload) $\mathrm{pmol} / \mathrm{g}$ of tissue (intergroup difference: NS). Such total numbers contrasted with those reported for normal hearts, which are about two-fold greater [13].

The functional properties of the transporters observed, in the obese rat hearts, under basal conditions, with insulin, or following increased workload are summarised by Table 2 . It should be recalled that in normal rat hearts, insulin has been shown to increase the Hill coefficient of plasma membranes glucose transporters

Table 1. Enzyme markers for enriched plasma and microsomal membranes obtained from hearts of genetically obese (fa/fa) rats perfused

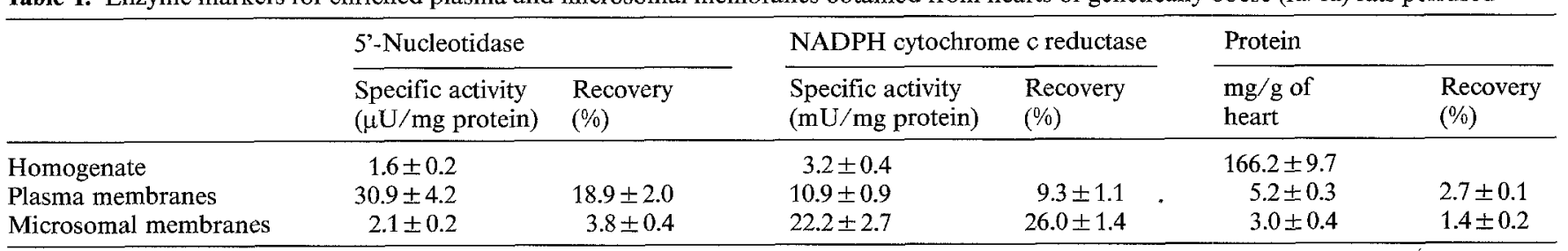

Hearts were perfused with Krebs Ringer bicarbonate buffer containing pyruvate $(5 \mathrm{mmol} / \mathrm{l})$. Membranes were purified as described in the Material and methods section. Results are means \pm SEM of duplicate determinations of three independent experiments 


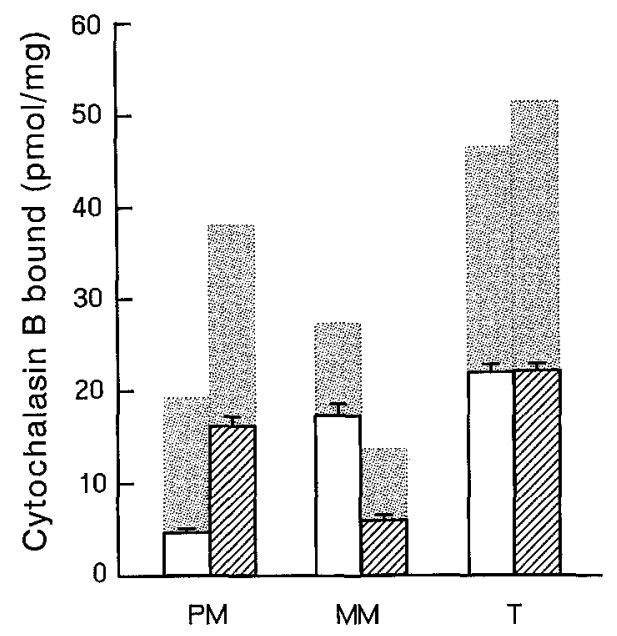

Fig.2. Effect of insulin $(10 \mathrm{mU} / \mathrm{ml})$ on the number of D-glucose transporters (measured by the specific binding of labelled cytochalasin $B$ ) in plasma (PM) and microsomal (MM) membranes from perfused obese rat hearts. Controls, $\square$, open bars; insulin, hatched bars. Total (T) refers to the number of glucose transporters present in both PM and MM expressed per mg protein. Each bar is the mean \pm SEM of 8 (controls) and 6 (insulin) individual experiments. Insulin effect on MM and PM glucose transporter number: $2 p<0.001$. Dotted areas : comparison

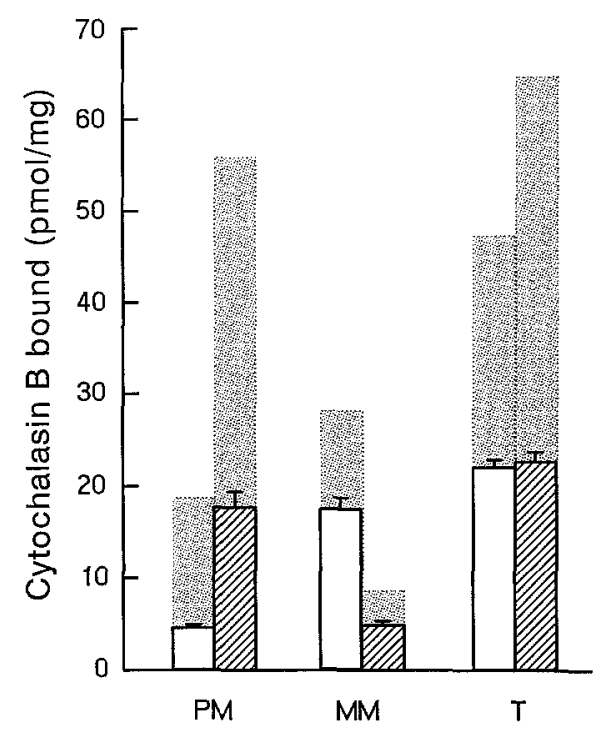

Fig.3. Effect of increased workload on the number of D-glucose transporters (measured by the specific binding of labelled cytochalasin B) in plasma (PM) and microsomal (MM) membranes from perfused obese rat hearts. Increased workload (hatched bars, Z) was achieved by raising perfusion pressure from $50 \mathrm{~mm} \mathrm{Hg}$ (controls, open bars, $\square$ ) to $100 \mathrm{~mm} \mathrm{Hg}$. Total (T) refers to the number of glucose transporters present in both PM and MM expressed per mg protein. Each bar is the mean \pm SEM of 8 (controls) and 6 (increased workload) individual experiments. Workload effect on MM and PM glucose transporter number: $2 p<0.001$. Dotted areas (四) are published data $[12,13]$ obtained in normal rat hearts, for comparison

(indicative of the occurrence of a positive cooperativity amongst transporters) and to increase the affinity of plasma membranes glucose transporters for cytochalsin B, hence possibly for glucose, thereby indirectly showing by these two changes that the hormone had in-
Table 2. Effect of insulin or increasing workload on glucose transporter Hill coefficient or constant of association (Ka) for cytochalasin B in plasma and microsomal membranes obtained from perfused hearts of genetically obese $(\mathrm{fa} / \mathrm{fa})$ rats

\begin{tabular}{llll}
\hline $\begin{array}{l}\text { Number of } \\
\text { experiments }\end{array}$ & $\begin{array}{l}\text { Control } \\
\text { (no stimulus) } \\
(8)\end{array}$ & $\begin{array}{l}\text { Insulin } \\
(6)\end{array}$ & $\begin{array}{l}\text { Increased } \\
\text { workload } \\
(6)\end{array}$ \\
\hline $\begin{array}{l}\text { Plasma membrane } \\
\quad \text { Transporter number (Ro) }\end{array}$ & $4.6 \pm 1.0$ & $16.1 \pm 1.0^{\mathrm{b}}$ & $17.6 \pm 1.6^{\mathrm{b}}$ \\
$\quad \begin{array}{l}\text { pmol/mg protein } \\
\text { Hill coefficient (n) }\end{array}$ & $3.0 \pm 0.2$ & $2.0 \pm 0.3^{\mathrm{a}}$ & $3.1 \pm 0.5$ \\
$\quad$ Ka (nmol/1) & $2.6 \pm 0.2$ & $3.1 \pm 0.4$ & $4.5 \pm 0.2^{\mathrm{a}}$ \\
$\begin{array}{l}\text { Microsomal membranes } \\
\text { Transporter number (Ro) }\end{array}$ & $17.3 \pm 1.2$ & $5.9 \pm 0.6^{\mathrm{b}}$ & $4.9 \pm 0.4^{\mathrm{b}}$ \\
pmol/mg protein & & & \\
$\quad$ Hill coefficient (n) & $3.1 \pm 0.2$ & $2.6 \pm 0.4$ & $3.2 \pm 0.1$ \\
Ka (nmol/1) & $4.6 \pm 0.3$ & $4.0 \pm 0.7$ & $4.0 \pm 0.1$ \\
\hline
\end{tabular}

Values are means \pm SEM of the number of experiments indicated. The Hill coefficient $(n)$ and the association constant $(\mathrm{Ka})$ were determined from the Hill analysis of the cytochalasin B- binding curves. Control: hearts perfused at $50 \mathrm{~mm} \mathrm{Hg}$; insulin, $10 \mathrm{mU} / \mathrm{ml}$; increased workload was achieved by raising perfusion pressure from 50 to $100 \mathrm{~mm} \mathrm{Hg}$. Plasma membrane data: ${ }^{\mathrm{a}} 2 p<0.01 ;{ }^{\mathrm{b}} 2 p<0.001$ when compared to control; when no indication $=$ NS. Microsomal membrane data: ${ }^{\mathrm{b}} 2 p<0.001$ when compared to control; when no indication $=$ NS

creased plasma membrane glucose transporters intrinsic activity $[12,13]$. In obese rat hearts and as shown in the present study (Table 2), these properties were altered: insulin paradoxically decreased the Hill coefficient and failed to increase the affinity $(\mathrm{Ka})$ values of plasma membrane glucose transporters. As also described before [13], workload in normal hearts has been shown to increase the Hill coefficient without, however, altering the affinity values of plasma membrane glucose transporters. In obese rat heart, workload failed to increase the Hill coefficient although it increased significantly the affinity values of plasma membrane glucose transporters for cytochalasin B. As in normal rat hearts $[12,13]$, the functional properties of the microsomal glucose transporters were unaffected by insulin or workload (Table 2).

The observation of a Hill coefficient of 3 under basal conditions was surprising, and suggested the possible occurrence of a positive cooperativity amongst transporters in the absence of stimuli. This could represent an adaptive phenomenon secondary to the observed decrease in transporter number (see Fig. 2 and 3). To ascertain this finding, 3-0-M-glucose uptake was measured at various concentrations of the hexose $(2-15 \mathrm{mmol} / \mathrm{l})$ : the analysis of the dose-response curve (not shown) also indicated that, under basal conditions, a Hill coefficient of 3 was found for actual 3-0-methyl D-glucose transport.

\section{Discussion}

The aim of the present work was to attempt defining some of the underlying pathological traits of the insulin-resistant heart of the genetically obese $(\mathrm{fa} / \mathrm{fa})$ rats. This was of importance as there are no data available 
for any of the many muscle types prone to become insulin-resistant.

It was observed that the basal, as well as the insulinor workload-induced increases in glucose uptake were diminished in perfused obese rat hearts, compared to that of lean rat (Fig. 1). These findings corroborate those of others made in other muscle types [4-6] and suggest that, although the heart is not representative of the muscle mass, it may undergo - upon the occurrence of insulin resistance - changes that are analogous to those seen in other muscles. This strengthens the observations made during the present study, with regard to the state of the glucose transporters as assessed by the cytochalasin B-binding assay. Indeed, it was found that the total number of glucose transporters present in obese rat hearts (Fig. 2 and 3) was decreased by about half compared to the values measured in normal rat hearts [13]. The translocation of transporters from an intracellular pool to the plasma membrane occurred in obese rat hearts, both under the influence of insulin or increasing workload. However, due to the decrease in the total number of glucose transporters, less of them were found (under basal conditions and after stimulation by insulin or workload) in the plasma membrane of the obese than in that of the lean rat hearts (Fig. 2 and 3).

Also, compared to normal plasma membrane glucose transporters that have been shown to be "activated" by insulin or workload $[12,13]$, obese rat plasma membrane transporters failed to be adequately "activated" by either one of the stimuli (Table 2).

In summary, the underlying pathology of the glucose transport in obese rat hearts (the first such report for a muscle type in insulin-resistant rodent) appears to be as follows: (a) the total number of transporters is decreased; (b) the translocation process triggered by stimuli is operative but, due to the prevailing decrease in the total number, it results in a lesser amount of transporters present within the plasma membrane; (c) the plasma membrane transporters are inappropriately "activated" by the stimuli considered. Insulin paradoxically decreased Hill coefficient without changing $\mathrm{Ka}$ values, whereas workload failed to increase Hill coefficient while increasing $\mathrm{Ka}$ values.

The precise etiology of the pathology just described has yet to be defined.

\begin{abstract}
Acknowledgements. This work was supported by Grant No 3.822.086, from the Swiss National Science Foundation (Berne, Switzerland) and by grant-in-aid from Nestlé S. A. (Vevey, Switzerland). The excellent secretarial work of Ms. K. Petermann and Ms. F. Touabi is gratefully acknowledged. We thank Mr. P. German for the computer-made graphs.
\end{abstract}

\section{References}

1. Jeanrenaud B, Halimi S, van de Werve G (1985) Neuroendocrine disorders seen as triggers of the triad: obesity-insulin resistanceabnormal glucose tolerance. Diabetes Metab Rev 1:261-291

2. Le Marchand-Brustel $Y$, Jeanrenaud B, Freychet $P$ (1978) Insulin binding and effects in isolated soleus muscle of lean and obese mice. Am J Physiol 234: E348-E358
3. Le Marchand-Brustel Y, Grémaux T, Ballotti R and van Obberghen E (1985) Insulin receptor tyrosine kinase is defective in skeletal muscle of insulin-resistant obese mice. Nature 315: 676-679

4. Crettaz M, Prentki M, Zaninetti D, Jeanrenaud B (1980) Insulin resistance in soleus muscle from obese Zucker rats. Involvement of several defective sites. Biochem J 186: 525-534

5. Zaninetti D, Crettaz M, Jeanrenaud B (1983) Dysregulation of glucose transport in heart of genetically obese $(\mathrm{fa} / \mathrm{fa})$ rats. Diabetologia 25: 525-529

6. Grundleger ML, Godbole VY, Thenen SW (1980) Age-dependent development of insulin resistance of soleus muscle in genetically obese (ob/ob) mice. Am J Physiol 239: E363-E371

7. Le Marchand-Brustel Y, Freychet P (1980) Alteration of glycogen synthase activation by insulin in soleus muscles of obese mice. Febs Lett 120: 205-208

8. Karnieli E, Zarnowski MJ, Hissin PJ, Simpson IA, Salans LB, Cushman SW (1981) Insulin-stimulated translocation of glucose transport systems in the isolated rat adipose cell. Time course, reversal, insulin concentration dependence, and relationship to glucose transport activity. J Biol Chem 256: 4772-4777

9. Suzuki K, Kono T (1980) Evidence that insulin causes translocation of glucose transport activity to the plasma membrane from an intracellular storage site. Proc Natl Acad Sci (USA) 77: 2542-2545

10. Klip A, Ramlal T, Young DA, Holloszy JO (1987) Insulin-induced translocation of glucose transporters in rat hindlimb muscles. Febs Lett 224: 224-230

11. Greco-Perotto R, Assimacopoulos-Jeannet F, Jeanrenaud B (1987) Insulin modifies the properties of glucose transporters in rat brown adipose tissue. Biochem $J$ 247: 63-68

12. Zaninetti D, Greco-Perotto R, Assimacopoulos-Jeannet F, Jeanrenaud B (1988) Effects of insulin on glucose transport and glucose transporters in rat heart. Biochem $\mathbf{J} 250: 277-283$

13. Zaninetti D, Greco-Perotto R, Jeanrenaud B (1988) Heart glucose transport and transporters in rat heart: regulation by insulin, workload and glucose. Diabetologia 31: 108-113

14. Hissin PJ, Foley JF, Wardzala LJ, Karnieli E, Simpson IA, Salans LB, Cushman SW (1982) Mechanism of insulin-resistant glucose transport activity in the enlarged adipose cell of the aged, obese rat. Relative depletion of intracellular glucose transport systems. J Clin Invest 70: 780-790

15. Hissin PJ, Karnieli E, Simpson IA, Salans LB, Cushman SW (1982) A possible mechanism of insulin resistance in the rat adipose cell with high fat/low carbohydrate feeding. Depletion of intracellular glucose transport systems. Diabetes 31: 589-592

16. Bradford M (1976) A rapid and sensitive method for the quantitation of microgram quantities of protein utilizing the principle of protein-dye binding. Anal Biochem 72:248-254

17. Avruch J, Wallach DFH (1971) Preparation and properties of plasma membrane and endoplasmic reticulum fragments from isolated rat fat cells. Biochem Biophys Acta 233: 334-347

18. Sottocasa GL, Kuylenstierna B, Ernster L, Bergstand A (1967) An electron transport system associated with the outer membrane of liver mitochondria. A biochemical and morphological study. J Cell Biol 32: 415-438

19. Greco-Perotto R, Zaninetti D, Assimacopoulos-Jeannet F, Bobbioni E, Jeanrenaud B (1987) Stimulatory effect of cold adaptation on glucose utilisation by brown adipose tissue. J Biol Chem 262: $7732-7736$

20. Hill AV (1910) The possible effects of the aggregation of the molecules of haemoglobin on its dissociation curves. J Physiol (London) $40: 190-229$

Received: 2 May 1988

and in revised form: 23 November 1988

Prof. B. Jeanrenaud

Laboratoires de Recherches Métaboliques

Avenue de la Roseraie 64

CH-1211 Geneva 4

Switzerland 